\title{
'I Have Something to Say': Supporting Aphasics for Organizing and Sharing Personal Experiences by Photos
}

\author{
Abdullah Al Mahmud and Jean-Bernard Martens \\ Department of Industrial Design, Eindhoven University of Technology \\ P.O. Box 513, 5600 MB Eindhoven, The Netherlands \\ \{a.al-mahmud, j.b.o.s.martens\} atue.nl
}

\begin{abstract}
When a person, due to brain injury or other disease, suffers in his or her ability to speak, it becomes inherently cumbersome to share needs, emotions, and experiences through personal stories and social interaction. We report on our early design exploration to share everyday experiences by photos for people having expressive aphasia. We also introduce the concept of a multimodal narrative template to help persons with aphasia to reconstruct their experiences and hence promote face-to-face communication and social interaction from everyday activities.
\end{abstract}

Keywords: Aphasia, Sharing experiences, Contextual interview.

\section{Introduction}

Much of our social life consists of sharing daily stories with other people. However, sharing personal stories can be extremely difficult for people with limited verbal ability, such as those suffering from expressive aphasia. As a result, aphasia often leads to increased social isolation and possibly to depression. Enabling persons with aphasia to share their daily experiences will help them to become more socially active and to re-engage with their preferred life style. Aphasia is an acquired communication disorder that is caused by brain injury or trauma. Aphasia affects language comprehension and generation [4], such that people's ability to express themselves verbally suffers. Speech Generating Devices (SGD) such as TouchSpeak [6] are widely used in aphasia therapy as well as during the post-therapy period though there are various limitations of SGDs such as (a) They contain isolated symbols whose meanings have to be learned and need to retrieve phrases or words to construct meaning which supports very simple stories. Therefore, these activities pose cognitive challenges for the users. (b) SGDs support needs-based interaction for functional communication. They lack the support for sharing experiences based on real-life events, which are key aspects to social interaction.

Sharing experiences from real-life events require evidence captured in different forms such as photos. Photographs have several functions such as preserving memories about the past, narrating and organizing everyday experiences, and communicating and building social relations [3]. Photography has been proposed within 'Aphasia Talks' [5] as a way of facilitating self-expression in persons with aphasia for reintegration, improving socialization and allowing recreation. The issue of how people with special needs can be empowered to use photos to share experience 
does not yet seem to have been explored in depth. A study has been reported to help persons with aphasia in building stories [2]. However, there were several limitations of the prototype such as the editing has to be done at the time of capture and lack of support for organizing the captured photos. Moreover, the story-building task was limited just by adding simple emoticons or sound and lacking the support to build a meaningful narrative for persons with aphasia. Therefore, our objective is to design a supporting system for sharing personal experiences by taking into account the limitations described above.

\section{Design Process}

Several design methods have been used to design for aphasics [1]. As a first step in the design process, it became essential to ascertain how aphasics and their partners are involved in the communication process. Moreover, we wanted to explore how photos could be used to share experience for aphasics and their communication partners.

Contextual Interview. We interviewed an aphasic (Gender: Male, Age: 65+) and his partner. He has been living with aphasia for seven years and he has problems in finding appropriate words and depends on his partner for confirmation. In response to the question of how the aphasic shared his past events, he said that he could not share due to his inability to express by words and lack of alternative support. Later on, his partner added that, he could not retell anything that he heard, saw or experienced. In such cases, captured photos could be helpful for sharing his experience. His partner also added, '...You would have used it if you were not able to communicate as you are now'. The aphasic also emphasized that it (i.e. sharing experience through photos) would be helpful for other aphasics who can barely speak.

Sharing Experience through Photos. The final part of the interview consisted of an experiment to explore how the aphasic would tell stories from photos. First, he was shown a single picture where an old woman was playing cards and we tried to find out what he would tell if he would show this picture to others. He expressed that if he finds that the other person ('who') in the picture is not familiar, he would tell that first and then 'what' he/she/it is doing. If the person or object displayed on the picture is familiar to him, then the most important thing is to tell 'what' it is or he/she is doing. When he used pictures that he was familiar with, he naturally started to narrate about these pictures such as who they were in the picture and how they were related to him. This was observed when he showed two pictures that were hanging on the wall, a picture of his partner and her sisters, and a picture of his partner and her tennis group. Therefore, providing personally relevant cues with 'who' and 'what' would help him to share if he was unable to find personal relevance of the pictures. Later on, a series of four pictures were shown in order to find out whether or not he would combine those pictures into a larger story. He reflected on the pictures separately, since he did not consider that there was a meaningful connection between them.

Insights from the Interview. The key implications are summarized below:

- Photos can be used to capture and report past events for aphasics. Identifying 'who' and 'what' property from photos would be the first step for building and narrating an experience. 
- Contextually rich image could invoke memories of the aphasics and consequently could help them to express their experiences. The contextually rich image should have clear personal association for aphasics.

- Support should be provided for 'why' and 'how' questions, that is why a picture has been taken and how it relates to a particular experience of aphasics.

Concept Design and Evaluation. We realized that it was not easy to intensively engage aphasics and elicit design requirements. Therefore, we did an experiment with a non-aphasic person to understand aphasic's experience and sharing capabilities. Our goal was to generate design requirements and later validate those with speech therapists. A voluntary non-aphasic participant was asked to capture daily experiences, later share it with us in a less familiar language, and act as if that he could only use his left hand for gesturing like aphasics. The experiment was done over two days. After the participant shared the experience in French (since he was less familiar with French language) from the captured photos, we expressed what we understood from what the participant told. The photos captured were related to his daily activities such as having lunch with friends, doing household work etc. It was observed that the participants had difficulties in expressing. He clustered the photos according to related activities and time to reflect each of them as a coherent story. The participant took in total eleven pictures that constituted two different stories. Since he was having problem in narrating the experience, he was given a template (template contains a grid such as who, where, what, emotion etc.) to help building the narrative. It was deduced that it was much easier for him to add relevant picture(s) for indicating and for us to understand the context as a whole story. The participant realized that he could take some more relevant pictures to express the right thing. However, next day he took ten pictures to tell three different stories. He also realized that he missed some joining phrases to tell experience from photos. It was also revealed that not many pictures were required to share the key daily experiences.

Based on the above findings we have designed an application that could help persons with aphasia to organize, build and share personal experiences from everyday photos. Firstly, the application helps to organize captured photos according to clusters on date and time to find out related events. The right side panel shows the most recent captured information (date and time) which is extracted from digital photos. Moreover, photos can be retrieved according to different clusters such as 'who', 'where' 'what'. Each of these clusters are subdivided into some basic categories such as 'who' cluster contains picture of family, friends and others. To help in building narrative of experiences a template is provided. The template has the option to fill appropriate picture according to 'who', 'where', 'what' and 'emotion property' (see Fig. 1). The template is a guided approach to construct a particular experience. If required the user can edit individual picture with text or inbuilt icons. There is an option for adding other media such as sound, video fragments if they are captured since these will enrich the understanding of the target experience by contextualizing it to others. In Fig 1, an example is shown where a narrative is constructed using the template. The relevant part of the template is filled to tell about the person, Pintu who is in the birthday party and the narrator is enjoying. This template helps to build photo driven or event driven narrative depending on the preference of users. 
Designing an appropriate interface for person with aphasia is challenging and needs iterations. We are still refining the conceptual prototype by evaluating it with speech therapists. Based on the feedback we will develop a working system. An appropriate capturing device will be designed to actively capture contextually rich images, which can easily be operated by one hand. The complete system (capturing, editing and sharing) will be evaluated in field tests with aphasics and their communication partner(s).

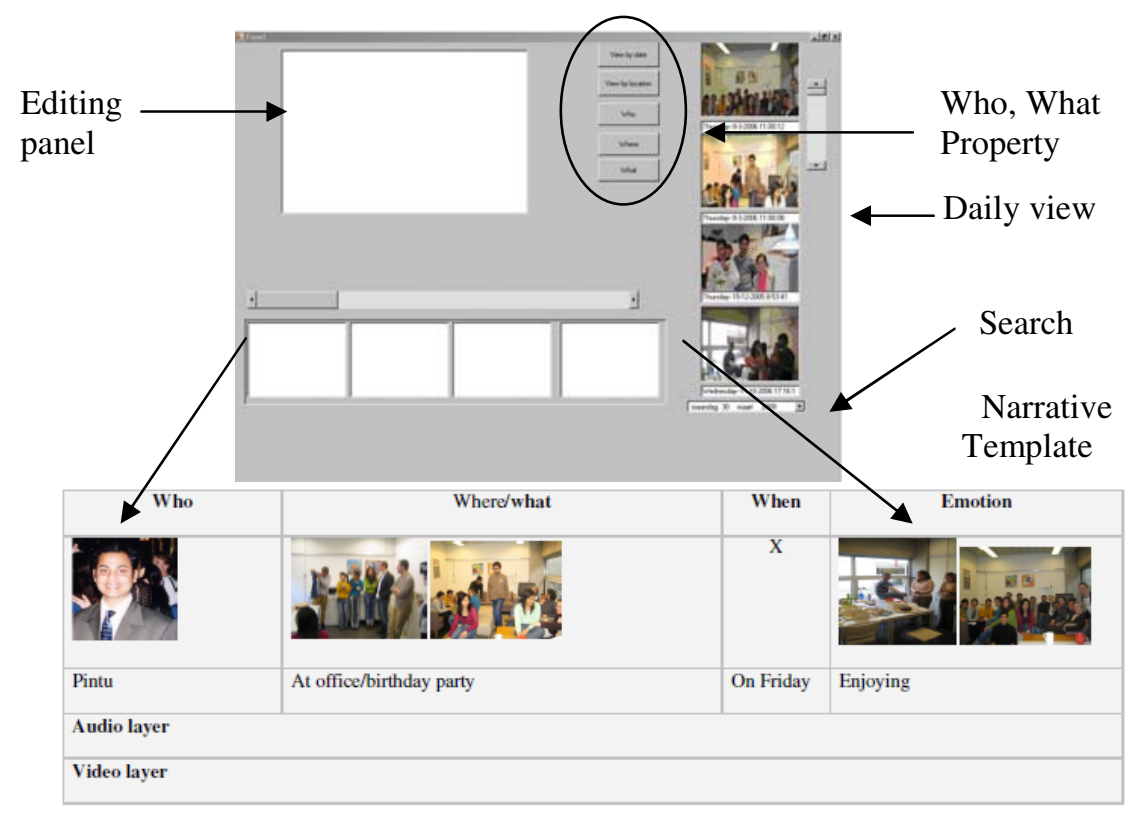

Fig. 1. Snapshot of the interface (top), a template to build a multimodal narrative of experiences (bottom)

\section{References}

1. Allen, M., McGrenere, J., Purves, B.: The Field Evaluation of a Mobile Digital Image Communication Application Designed for People with Aphasia. ACM Trans. Access. Comput. 1(1), 1-26 (2008)

2. Daemen, E., Dadlani, P., Du, J., Li, Y., Erik-Paker, P., Martens, J.B., de Ruyter, B.: Designing a free style, indirect, and interactive storytelling application for people with aphasia. In: Baranauskas, C., Palanque, P., Abascal, J., Barbosa, S.D.J. (eds.) INTERACT 2007. LNCS, vol. 4662, pp. 221-234. Springer, Heidelberg (2007)

3. Harrison, B.: Photographic visions and narrative inquiry. J. Narrative Inquiry $12(1), 87-111$ (2002)

4. Hillis, A.A.: Progress in the last quarter of a century. Neurology 69, 200-213 (2007)

5. Levin, T., Scott, B., Borders, B., Hart, K., Lee, J., Decanini, A.: Aphasia Talks: photography as a means of communication, self-expression, and empowerment in persons with aphasia. J. Topics in stroke rehabilitation 14(1), 72-84 (2007)

6. TouchSpeak, http://www. touchspeak.co.uk/ 